\title{
Philosophiques
}

\section{La documentation en philosophie : bibliographies courantes}

\section{Gilles Paradis}

Volume 6, numéro 1, avril 1979

URI : https://id.erudit.org/iderudit/203114ar

DOI : https://doi.org/10.7202/203114ar

Aller au sommaire du numéro

Éditeur(s)

Société de philosophie du Québec

ISSN

0316-2923 (imprimé)

1492-1391 (numérique)

Découvrir la revue

Citer cet article

Paradis, G. (1979). La documentation en philosophie : bibliographies courantes. Philosophiques, 6(1), 177-199. https://doi.org/10.7202/203114ar d'utilisation que vous pouvez consulter en ligne.

https://apropos.erudit.org/fr/usagers/politique-dutilisation/ 


\title{
LA DOCUMENTATION EN PHILOSOPHIE : BIBLIOGRAPHIES COURANTES
}

\author{
par Gilles Paradis
}

Si Pic de la Mirandole revenait parmi nous et s'il était désireux de s'informer sur tout ce qui se publie en philosophie, il aurait sûrement fort à faire. En 1977, par exemple, le Répertoire bibliograpbique de la pbilosopbie signalait 10,360 titres. Cette croissance de la production philosophique s'inscrit à l'intérieur du phénomène plus large de l'explosion documentaire qui a touché la plupart des disciplines depuis quelques années.

Pour repérer la documentation requise, il devient dès lors nécessaire d'avoir recours à des instruments de consultation conçus de telle sorte qu'ils nous permettent d'extraire de la production philosophique totale les publications qui nous intéressent plus particulièrement. Parmi ces instruments de consultation, on compte les bibliographies courantes destinées à répertorier régulièrement les nouvelles parutions dans une discipline donnée.

En philosophie, nous disposons de plusieurs bibliographies courantes et il sera utile, dans un premier temps, d'en dresser le bilan et de décrire les principales. Nous ajouterons, dans la seconde partie, quelques commentaires sur le contrôle bibliographique en philosophie et sur les perspectives ou tendances qui se dessinent à l'heure actuelle dans ce domaine.

Avant d'aborder notre recherche, il convient d'apporter quelques précisions sur son objet même. Par bibliographie courante, nous entendons ici un ouvrage de consultation qui paraît à intervalles plus ou moins réguliers et qui vise principalement et de façon systématique à faire connaître les nouvelles publications en philosophie. Notre étude ne comprend donc pas les revues philosophiques qui possèdent une 
section de comptes rendus, un bulletin spécialisé comme on en trouve dans la Revue des sciences philosophiques et théologiques ou encore une bibliographie d'ouvrages récemment reçus chez l'éditeur de la revue.

Par ailleurs nous nous en tenons uniquement aux bibliographies courantes qui paraissent encore de nos jours. Sont donc exclus ici les ouvrages de référence tels que Pbilosopbic Abstracts ou Scripta Recenter Edita qui paraissaient régulièrement mais dont la publication a été suspendue. On trouvera une liste de ces bibliographies courantes dans Periodica Pbilosophica ${ }^{1}$ de W. Hogrebe, R. Kamp et G. Köning, dans Bibliography of Philosophical Bibliographies ${ }^{2}$ de $\mathrm{H}$. Guerry ainsi que dans $A$ History of the Bibliography of Philosophy ${ }^{3}$ de M. Jasenas.

Parmi les bibliographies courantes qui paraissent actuellement, les unes recensent les publications de plusieurs pays alors que d'autres ont davantage un caractère national. Quelques bibliographies enfin signalent et analysent de façon critique un nombre limité d'ouvrages. Même si cet ordre n'est pas strict, nous allons l'emprunter ici dans l'énumération des divers instruments documentaires.

\section{1) BILAN DESCRIPTIF DES BIBLIOGRAPHIES COURANTES EN PHILOSOPHIE}

\section{a) Le Répertoire bibliographique de la philosopbie}

Le Répertoire bibliographique de la philosopbie 4 paraît comme publication autonome et sous sa forme actuelle depuis 1949. Son histoire toutefois remonte au-delà de cette date et est étroitement liée à l'existence de la Revue philosophique de Louvain. C'est en effet comme supplément ou en annexe à cette revue que le répertoire a vu le jour à la fin du siècle dernier. Rappelons en passant que la Revue philosopbique de Louvain porte

1. Hogrebe Wolfram, Kamp, Rudolf et Köning, Gert, Periodica Philosophica, Düsseldorf, Philosophia Verlag, 1972.

2. GUerry, Herbert, A Bibliography of Philosophical Bibliographies, Wesport, Connecticut, Greenwood Press, 1977

3. JASENAS, Michael, A History of the Bibliograpby of Pbilosophy, Hildesheim, Georg Olms Verlag, 1973.

4. Répertoire bibliographique de la philosophie, Louvain, Institut Supérieur de Philosophie, v. 1, $1949-$ 
ce nom depuis 1946. Auparavant, soit depuis 1910, elle s'appelait la Revue néo-scolastique de philosophie et de 1894 à 1909 elle a eu pour titre la Revue néo-scolastique.

Pour l'Institut Supérieur de Philosophie de Louvain, l'idée de dresser la liste des ouvrages et des revues de philosophie remonte à 1895 alors que la Revue néo-scolastique commença à éditer en annexe un «Sommaire idéologique des ouvrages et des revues de philosophie $»$. Ce sommaire paraissait chaque trimestre et les publications étaient regroupées selon la classification décimale de Melvil Dewey. L'entreprise se continua jusqu'en 1914. Elle fut interrompue de 1915 à 1933. En mai 1934 la Revue néo-scolastique de philosophie publiait en supplément la première livraison du Répertoire bibliographique. Jugeant que la classification de Dewey ne présentait pas d'avantages décisifs, les éditeurs abandonnèrent cette méthode de classification au profit d'un arrangement des publications qui ressemble à ce que nous connaissons aujourd'hui. La publication du répertoire se prolongea jusqu'en mai 1940, fut interrompue durant la guerre puis reprise au mois d'août 1946. À partir de 1949 , le répertoire devint une publication autonome qui paraît depuis lors sous sa forme actuelle.

Soulignons que le Répertoire bibliographique est publié simultanément depuis 1939 comme supplément à la revue néerlandaise Tijdschrift voor Philosophie. Comme la publication de cette revue n'a pas cessé durant la seconde guerre mondiale, c'est là qu'on peut trouver la bibliographie courante pour cette période. Encore faut-il se rappeler que la bibliographie rétrospective de G.A. De $B_{r i e}^{5}$ couvre, entre autres, cette époque.

C'est I'Institut Supérieur de Philosophie de l'Université de Louvain qui est, encore de nos jours, responsable de la publication trimestrielle du répertoire. La publication est en outre réalisée sous les auspices de l'Institut International de Philosophie et avec le patronage de l'Unesco.

Le répertoire se donne pour tâche de signaler les livres et les articles de périodiques publiés dans les principales langues

5. De Brit, G.A., Bibliograpbia Pbilosopbica 1934-1945, Bruxelles, Éditiones Spectrum, 1950,2 volumes. 
occidentales soit l'allemand, l'anglais, l'espagnol, le catalan, le français, l'italien, le néerlandais et le portuguais. Occasionnellement, on retrouve des publications appartenant à une autre langue. Pour les langues occidentales le répertoire cherche donc à être exhaustif alors qu'il est sélectif pour les autres langues. Plus de quatre cents périodiques sont dépouillés régulièrement.

D'autre part, le répertoire est purement signalétique ; il nous fournit donc la référence complète pour chaque publication mais sans analyse de contenu. Le répertoire se limite en outre à la littérature philosophique, cette expression étant prise en un sens assez strict: par exemple en psychologie, le répertoire se borne à mentionner les ouvrages généraux ou les publications relatives à la méthode et à la critique de cette science.

Il n'empêche que le répertoire compte maintenant plus de 10,000 entrées chaque année. Un même ouvrage peut toutefois se retrouver sous une ou plusieurs rubriques. Ces données bibliographiques sont comprises dans les trois premiers fascicules de l'année. Le quatrième fascicule, publié en novembre, recense les comptes rendus contenus dans les revues dépouillées par le répertoire.

La bibliographie courante de Louvain se divise en deux parties principales. D'abord une section historique qui regroupe selon l'ordre chronologique de la date de naissance des philosophes les œuvres de ces philosophes ou les études qui s'y rapportent. Cette section comporte 33 divisions ou subdivisions. Dans la seconde partie, composée de 61 divisions ou subdivisions, les ouvrages sont réunis selon les grandes branches de la philosophie.

Numériquement, la section historique est plus importante. En 1976, par exemple, 60\% des publications se retrouvaient dans la première partie contre $40 \%$ pour la seconde. Repérables dans la partie systématique, ces publications le sont aussi à l'aide de la table onomastique et de la table des anonymes. La première table signale les publications d'un auteur ou les études qui s'y rapportent. La table des anonymes permet de retrouver les ouvrages non directement 
repérables par le nom d'un auteur, comme c'est le cas pour les actes d'un congrès.

Enfin le délai entre la publication d'un ouvrage ou d'un article et sa mention dans le répertoire varie beaucoup. Ce délai s'étend de quelques mois à un an ou deux, parfois davantage.

\section{b) La Bibliographie de la philosophie}

La Bibliographie de la philosophie ${ }^{6}$ est publiée grâce aux efforts conjugés de l'Institut International de Philosophie, de l'UNESCO, du Centre National de la Recherche Scientifique de Paris et du Centre de Documentation et de Bibliographies Philosophiques de l'Université de Besançon. Sous sa forme actuelle, la Bibliographie de la philosopbie paraît depuis 1954. Elle a été précédée d'une bibliographie du même nom, publiée de 1937 à 1953, mais dont la conception différait à plusieurs points de vue.

Cette première formule de la Bibliographie de la philosophie a été éditée deux fois l'an, sauf durant la période comprise entre juillet 1939 et décembre 1945. En outre, on compte un seul volume pour 1950 et un seul volume aussi pour 1951. Les années 1952 et 1953 ont pour leur part été réunies.

Sous sa forme première, la bibliographie répertoriait les livres publiés dans une quarantaine de pays et les articles de 700 revues environ. La bibliographie comprenait en outre plusieurs sections : d'abord une liste des éditeurs regroupés par pays, suivie d'une liste des revues organisée de la même manière. Puis venait la partie principale, soit le recensement des livres et des articles (et quelquefois des thèses de doctorat) distribués selon l'ordre alphabétique des auteurs. La section suivante reprenait plusieurs publications, mais en les regroupant selon l'ordre chronologique ou géographique. La cinquième section était constituée d'un index des philosophes et des savants, et la sixième d'un index des concepts philosophiques inspiré du dictionnaire de Lalande.

6. Biblingraphie de la philosophie, publié par I'Institut International de Philosophie, Paris, Vrin, vol. 1-10, 1937-1953; vol. 1, 1954- 
À partir de 1954, la Bibliograpbie de la philosopbie devient trimestrielle et elle se limite alors à inventorier les livres dont elle commence cependant à fournir un résumé. Ce changement s'explique par la volonté des éditeurs d'éviter le double emploi, d'une part, avec le Répertoire bibliographique de la philosophie dont le mandat couvre le signalement des livres et des articles de revues et, d'autre part, avec le Bulletin signalétique axé sur les revues.

Présentement chaque pays participant, via son centre de bibliographie philosophique, envoie à l'Institut International de Philosophie les nouvelles publications avec une analyse de leur contenu. Le résumé sans appréciation critique est signé par un spécialiste. Ce résumé n'excède pas 400 mots et il est rédigé en français, anglais, allemand, italien ou espagnol quand l'ouvrage appartient à l'une de ces langues, et en français ou en anglais dans les autres cas. Les traductions, les rééditions et les réimpressions sont habituellement signalées à la fin de chaque chapitre, mais sans résumé. On trouve plutôt dans ces cas quelques explications bibliographiques.

Selon les éditeurs, " la Bibliographie de la philosophie est exhaustive de droit. Que ce but soit atteint, dépend néanmoins de l'envoi régulier, par les auteurs et les éditeurs, des informations suffisantes à leur «Centre National " respectif ${ }^{7}$. Le nombre moyen annuel des entrées depuis 1954 s'élève à 1250 , ce chiffre incluant les diverses entrées pour un même ouvrage. En 1977, d'autre part, la table des éditeurs comprenait 19 pays, plus de la moitié de ces pays étant européens.

La bibliographie est répartie en dix grandes sections qui représentent autant de branches de la philosophie. À l'intérieur de chaque section, les ouvrages sont mentionnés selon l'ordre alphabétique des auteurs, sauf dans le cas de la neuvième section qui est consacrée à l'histoire de la philosophie et qui adopte plutôt l'ordre chronologique.

Le dernier numéro de l'année inclut trois index. Le premier dresse la liste des auteurs, des sujets et des titres des

7. Bibliographie de la philosophie. Avertissement. 
ouvrages qui ont fait l'objet d'une analyse ; les sujets sont tirés du titre et quelquefois d'un terme significatif du résumé. Un deuxième index mentionne les préfaciers, les traducteurs, les éditeurs et les philosophes cités. La troisième table signale les maisons d'édition par pays.

Le délai entre la parution d'un livre et son signalement dans la Bibliographie de la philosophie varie entre six mois et deux ans, parfois plus.

\section{c) Le Bulletin signalétique}

Le Bulletin signalétique ${ }^{8}$ est édité par le Centre National de la Recherche Scientifique à Paris par l'entremise de son Centre de documentation en sciences humaines. La publication du Bulletin signalétique remonte à 1940 . Il portait alors le titre de Bulletin analytique, appellation qu'il conserva jusqu'en 1955. C'est seulement en 1947 toutefois que le bulletin consacra une section à la philosophie. Pendant un bon moment cette section de philosophie fut reliée à d'autres disciplines comme la sociologie, la psychologie ou encore les sciences religieuses. Depuis 1970 cependant, le fascicule réservé à la philosophie est publié séparément des fascicules des autres disciplines et il porte le numéro 519.

Publié quatre fois l'an, le Bulletin signalétique recense les articles de revues philosophiques et les articles d'autres revues susceptibles de présenter un intérêt pour la philosophie. Le bulletin signale également les actes de congrès et de colloques, les volumes de mélanges et les rapports.

Le Centre de documentation en sciences humaines reçoit et dépouille annuellement quelque 1200 revues traitant en tout ou en partie de philosophie, ce qui constitue un important fonds documentaire de périodiques. Un fascicule trimestriel recense les articles de 320 revues environ.

Au cours des six dernières années, le bulletin a totalisé en moyenne 5,500 entrées chaque année. Cette documentation est depuis 1973 répartie en 12 grandes classes. La première classe

8. Bulletin signalétique 519: Philosophie, Paris, Centre National de la Recherche Scientifique, vol. 1, 1947- 
signale les études historiques. Cette section qui regroupe un peu moins de la moitié de la documentation constituait autrefois la dernière partie du bulletin. De plus, cette partie historique est maintenant divisée selon l'ordre alphabétique des philosophes, non par ordre chronologique. Les onze autres sections contiennent les études doctrinales (métaphysique, philosophie de l'art et ainsi de suite). Les 12 sections réunies forment la partie "Analyses et signalements" du fascicule. C'est là que nous retrouvons les données bibliographiques (auteur, titre, source, ...) ainsi qu'un résumé en français de l'article qui nous présente une idée du contenu sans appréciation critique. Ce résumé comprend habituellement entre 30 et 150 mots.

L'intérêt du Bulletin signalétique tient en outre à ce qu'il contient une table des concepts nous permettant de retrouver la documentation par le biais d'un sujet spécifique. La table des concepts indique en effet dans quel contexte le terme est employé, par exemple :

Causalité - Liberté - Schelling

Causalité - Quatre types - Aristote

Il existe aussi une table des auteurs des articles incluant les auteurs d'ouvrages pour lesquels le bulletin signale un compte rendu. Toutefois le signalement des comptes rendus est plutôt occasionnel et il se limite aux recensions élaborées. L'index des concepts et la table des auteurs sont cumulés annuellement.

D'autre part, le bulletin indique si le document est conservé au Centre de documentation en sciences humaines du CNRS ou dans l'une des 37 autres bibliothèques dont la liste apparaît au début de chaque fascicule. En outre, moyennant certaines conditions, il est possible d'obtenir une reproduction (photocopie ou microfiche) du document désiré.

Notons enfin que la production du Bulletin signalétique fait appel aux procédés automatiques pour la photocomposition, le traitement des données et même le repérage de la documentation grâce au système P.A.S.C.A.L. (Programme Appliqué à la Sélection et à la Compilation Automatique de la Littérature). Le Programme se divise en deux parties soit 


\section{P.A.S.C.A.L./Sciences et techniques et P.A.S.C.A.L./Sciences humaines.}

En philosophie, le Programme P.A.S.C.A.L. offre trois profils standards qui portent sur Wittgenstein, Aristote et Marx. L'abonnement à un profil standard permet de recevoir à intervalles de six semaines des fiches cartonnées sur les philosophes en question. Il est par ailleurs possible d'effectuer en différé des recherches rétrospectives dans le secteur des sciences humaines et sociales, cela à partir de la bibliographie de 1973.

\section{d) The Pbilosopher's Index}

Parmi les bibliographies courantes de caractère international en philosophie, le Pbilosopher's Index ${ }^{9}$ est le seul instrument de consultation qui n'est pas publié en Europe. C'est en effet le Philosophy Documentation Center de l'Université Bowling Green aux États-Unis qui est responsable de la publication trimestrielle et de l'édition cumulative annuelle du Philosopher's Index.

Cet index dépouille les revues - une quarantaine en 1967 et plus de 280 maintenant. Les éditeurs ont retenu les principales revues philosophiques de langue anglaise, française, allemande, espagnole et italienne, mais ils ont été plus sélectifs dans le cas des périodiques publiés dans d'autres langues ou dans le cas de revues interdisciplinaires reliées à la philosophie. Plus de la moitié des revues répertoriées sont de langue anglaise.

L'intérêt du Pbilosopher's Index provient de ce que la documentation signalée est accessible par un index des sujets généraux mais aussi particuliers. Car si nous retrouvons dans l'index des sujets les grandes branches de la philosophie, nous remarquons également des sujets particuliers tels que l'ironie, le paradoxe du menteur, l'opinion, les nombres, en plus des philosophes, des périodes historiques et des philosophies nationales. En fait, chaque article est lu par un philosophe professionnel qui choisit 5, 6 ou 7 descripteurs pour en rendre

9. The Philosopher's Index; an International Index to Philosophical Periodicals, Ohio, Bowling Green University, Philosophy Documentation Center, vol. 1, 1967- 
compte. Ces descripteurs deviennent autant de voies d'accès à la documentation.

Un deuxième index regroupe les articles par ordre alphabétique des noms d'auteurs. Dans cette section, nous retrouvons, en plus du nom de l'auteur, le titre de l'article, la référence complète et un résumé destiné à nous présenter une idée du contenu de l'article. Les résumés ont été ajoutés à partir de 1969, mais encore aujourd'hui plusieurs articles n'en comportent pas. Il faut dire que ces résumés proviennent le plus souvent de l'auteur lui-même. Les éditeurs dépendent donc de la collaboration plus ou moins diligente d'un nombre considérable de personnes. Pour les revues de langue allemande, le résumé des articles est obtenu par l'entremise de l'Institut philosophique de l'Université de Dusseldorf.

La troisième section du Philosopher's Index recense chaque année environ 1500 comptes rendus d'ouvrages récents. Une autre section mentionne quelques traductions parues ou en cours de préparation et notamment celles qui sont signalées par le Translation Center of Southern Illinois University.

D'autre part, il faut compter de 3 à 6 mois avant qu'un article soit mentionné dans le Philosopher's Index. Dans certains cas cependant le délai excède un an.

Le Philosopher's Index est produit, en partie du moins, à l'aide de l'informatique. C'est ainsi que les données sont entrées en mémoire d'ordinateur, et les différents index sont organisés et imprimés selon des programmes déterminés. Il n'en demeure pas moins que l'indexation et la composition du résumé relèvent de l'intervention humaine. Ce choix s'explique d'abord, selon Richard H. Lineback ${ }^{10}$, par des raisons financières. L'indexation automatique s'avère en effet plus coûteuse que l'indexation manuelle. L'indexation manuelle comporte en outre quelques avantages. Elle permet d'attribuer un descripteur aux termes qui apparaissent peu fréquemment dans l'article mais qui sont révélateurs de son contenu. En utilisant un court texte plutôt qu'une série de mots-clefs, l'indexation manuelle permet aussi d'indiquer plus aisément la conclusion.

10. Lineback, Richard H., The Philosopher's Index, dans CIRPHO, vol. 2, 1974, $\mathrm{n}^{\circ} 1, \mathrm{p}$. 19-20. 
Soulignons enfin que depuis quelque temps déjà le Philosophy Documentation Center a rendu public son projet de rendre le Pbilosopher's Index accessible par ordinateur ${ }^{11}$. En 1978, il était possible d'interroger le Pbilosopher's Index par ordinateur mais en différé.

\section{e) Bibliographie Philosophie}

La Bibliographie Philosophie ${ }^{12}$ est un répertoire bibliographique produit à l'aide de l'ordinateur sous la responsabilité de l'Institut für Gesellschaftswissenchaften und Dokumentation (Berlin). La bibliographie mentionne la production philosophique mondiale disponible en République Démocratique Allemande. Sont signalés les livres, les recensions d'ouvrages, les volumes de mélanges et les articles de plus de 350 périodiques. Les thèses toutefois ne sont pas recensées. Par ailleurs l'accent est mis sur la philosophie marxiste, comme en témoigne la classification décimale utilisée dans le répertoire. Cette classification regroupe en effet les publications sous deux rubriques générales: la philosophie marxiste (classes 100 à 189) et la philosophie non marxiste (classes 190 à 199). Un index des auteurs et un index des sujets généraux et spécifiques facilitent la consultation de la bibliographie.

D'autre part, deux suppléments sont parus qui fournissent des informations additionnelles sur l'utilisation de la bibliographie. Le premier supplément comprend les divisions et les subdivisions de la classification utilisée dans cette bibliographie, la liste et le code des bibliothèques qui possèdent certaines publications mentionnées dans la bibliographie ainsi que la liste des revues dépouillées. En ce qui a trait aux nouvelles revues dépouillées, elles sont mentionnées dans les numéros courants. Le second supplément constitue, en fait, un thesaurus fournissant les descripteurs et les subdivisions de forme utilisés dans la classification.

\footnotetext{
11. À ce sujet voir l'article de Richard H. LINEBACK, "The Philosopher's Information Retrieval System ", dans Revue internationale de philosopbie, vol. 27, 1973, fasc. I, p. 95-104.

12. Bibliographie Pbilosophie, Berlin, Zentralstelle für die philosophische Information und Dokumentation im Institut für Gesellschaftswissenchaften beim ZK der SED, 1967 -
} 
f) Bibliografia filosofica italiana

Le Centro di Studi Filosofici Cristiani di Gallarate est l'organisme qui publie le Bibliografia filosofica italiana' ${ }^{13}$. Depuis 1949, un volume est consacré chaque année à la recension des publications italiennes, monographies et articles de revues. Plus de 140 revues appartenant à la philosophie ou à une discipline connexe sont dépouillées régulièrement. La bibliographie signale en outre les recensions d'ouvrages : dans la partie principale pour les livres italiens, en appendice pour les livres en langue étrangère mais édités en Italie.

L'organisation interne de la bibliographie ressemble à celle du Répertoire bibliographique de la philosophie. Les deux principales sections regroupent les publications sous le titre Histoire de la philosophie et Philosophie. Dans le premier cas, l'ordre est chronologique, alors que dans le second les publications sont réunies selon les grandes divisions de la philosophie. Un index des auteurs facilite la consultation de l'ouvrage.

La Bibliografia filosofica italiana constitue donc une bibliographie nationale assez complète et signalétique. Deux ouvrages rétrospectifs se sont ajoutés à ce travail périodique. Un premier ouvrage comprend les publications philosophiques italiennes parues de 1850 à $1900^{14}$. Le second ouvrage publié en quatre tomes couvre la période de 1900 à $1950^{15}$. Dans les deux cas, les œuvres des philosophes ou les études s'y rapportant sont signalées selon l'ordre alphabétique des philosophes. Soulignons aussi que les revues philosophiques italiennes qui paraissaient durant ces deux demi-siècles font l'objet d'une rubrique descriptive élaborée.

g) Bibliografia Filosofica Espanôla ${ }^{16}$

La littérature philosophique espagnole est répertoriée

\footnotetext{
13. Bibliographia Filosofica Italiana, Centro di Studi Filosofici di Gallarate, Padova, Editrice Gregoriana, vol. 1, 1949-

14. Insrituro di Studi Filosofica, Bibliographia filosofica italiana 1850-1900, Roma, Edizione Abete, 1969.

15. Instituto di studi filosofici e del Centro Nazionale di Informazioni Bibliografiche, Bibliografia filosofica italiana dal 1900 al 1950, Roma, Edizione Delfino, 1950-1956, 4 volumes.

16. Bibliografia Filosofica Espãnola, dans Pensamiento, Madrid, 1945-
} 
chaque année depuis 1945 dans la revue Pensamiento publiée à Madrid. De 700 à 800 livres ou articles de revues édités en Espagne, au Mexique, au Venezuela ou dans d'autres pays de langue espagnole sont recensés régulièrement. La bibliographie adopte la division fréquente entre la partie historique et la partie systématique. La première partie est divisée selon les périodes historiques et subdivisée par auteurs. La seconde comprend une section pour la métaphysique, l'esthétique et les autres branches de la philosophie.

Luis Martinez Gomez a travaillé pendant de nombreuses années à la publication de cette bibliographie philosophique. Il est aussi l'auteur de la Bibliografía filosófica espanôla $e$ hispanoamericana (1940-1958) ${ }^{17}$, ouvrage qui cumule entre autres les publications déjà signalées dans la revue Pensamiento pour ces années. Cette bibliographie recense plus de 10,000 titres.

\section{h) Bibliografia filosófica mexicana}

La Bibliografía filosófica mexicana ${ }^{18}$ est une bibliographie annuelle et signalétique qui recense les livres, les articles d'une vingtaine de revues, les comptes rendus d'ouvrages et les thèses publiés au Mexique dans le champ de la philosophie. Chaque fascicule annuel comprend quelques centaines de titres. Par exemple, le répertoire qui couvre l'année 1971 et qui a été publié en 1974 regroupe 330 publications. Le cadre de classement est celui-là même que l'on retrouve dans les bibliographies courantes en philosophie, soit l'histoire et les grandes branches de la philosophie. Pour faciliter la consultation, la bibliographie est pourvue d'un index incluant les auteurs et aussi les philosophes dont le nom apparaît dans le titre des publications.

En plus de la bibliographie courante, on trouve souvent dans ce répertoire des bibliographies spéciales. Le numéro 2, publié en 1971, fournit la bibliographie de José Gaos (1900-1969); le numéro 3, édité en 1973, présente une

17. Martinez Gomez, L., Bibliografia filosofica española e bispanoamericana, 1940-1958, Barcelona, J. Flors, 1961.

18. Bibliografia Filosófica Mexicana, 1968- , Mexico, Universidad Nacional Autonoma de Mexico, Instituto de Investigaciones Filosoficas, 1970- 
bibliographie du Dr. Eduardo Garcia Maynez et le numéro 4, paru en 1974, se termine par l'index de la Revista Filosofia y Letras pour les années 1941 à 1958.

\section{i) Handbook of Latin American Studies}

Le Handbook. of Latin American Studies ${ }^{19}$ vise à signaler annuellement les ouvrages publiés en Amérique latine ou concernant cette région. La sélection des ouvrages et les annotations sont assurées par un groupe de spécialistes. Le répertoire qui a commencé à paraître en 1936 pour l'année 1935 couvre plusieurs disciplines. Depuis 1964, le Handbook of Latin American Studies se divise en deux sections qui paraissent de façon alternative ; les Humanités d'une part et les Sciences sociales d'autre part.

La partie consacrée à la philosophie a été introduite avec le numéro 5 (1939). On la retrouve de nos jours dans la section des Humanités. Les livres et les articles de philosophie de plus de 60 revues sont signalés et annotés. On compte pour un volume de 200 à 300 entrées ayant trait à la philosophie. Ces publications sont regroupées sous une quinzaine de classes (histoire de la philosophie, esthétique, métaphysique, etc.). Chaque classe est subdivisée par ordre alphabétique des auteurs des publications.

Il existe un index des auteurs et un index des sujets. En outre un index cumulatif des auteurs couvre les années 1936-1966 (numéros 1-28).

j) Bibliography of Soviet Philosophy. Bibliographie der Sowjetischen Pbilosophie 20

C'est dans le périodique Studies in Soviet Thought que l'on retrouve maintenant la bibliographie courante des publications soviétiques (livres et articles de revues) à caractère philosophique. La bibliographie ne paraît pas dans chaque numéro de la revue, mais un système de numérotation qui tient compte de l'année et du fascicule de la revue permet de se retrouver

19. Handbook of Latin American Studies, Gainesville, University of Florida, vol. 1, 1935-

20. Bibliography of Soviet Pbilosophy. Bibliographie der Sowjetischen Pbilosophie, dans Studies in Swiet Thought, vol. 7, $\mathrm{n}^{\circ}$ 3, 1967- 
aisément. Les ouvrages sont en outre mentionnés selon l'ordre alphabétique des auteurs. Un index des auteurs et un index des sujets paraissent de façon alternative.

Studies in Soviet Thought est publié à l'Université de Fribourg en Suisse par l'Institute of East European Studies. C'est le même organisme qui avait pris l'initiative d'éditer, dans la collection Sovietica, la Bibliography of Soviet Pbilosophy pour les années 1947-196621. Cette bibliographie rétrospective d'une vingtaine d'années comprend sept tomes et 13,823 entrées. En fait, la bibliographie courante contenue dans le Studies in Soviet Thought continue le travail déjà amorcé dans la bibliographie rétrospective.

Dans le cas de la bibliographie courante, comme dans celui de la bibliographie rétrospective, on a prévu la translitération du russe et les index de sujets sont en langue anglaise.

k) Philosophical Books

Pbilosophical Books ${ }^{22}$ paraît trois fois l'an. Cette revue présente des comptes rendus descriptifs et critiques d'une sélection d'ouvrages récents publiés en langue anglaise. Chaque numéro comporte 20 à 25 analyses signées par des philosophes professionnels. Le comité de rédaction se compose de membres qui pour la plupart sont impliqués dans la publication de la revue Analysis. Depuis quelques années, les recensions d'ouvrages sont rangées sous la rubrique Histoire de la philosophie ou Philosophie contemporaine avec une subdivision par ordre alphabétique des auteurs.

\section{1) Pbilosopby and History}

Pbilosopby and History ${ }^{23}$ est une publication semi-annuelle qui paraît dans la collection German Studies depuis 1968. La revue comprend, comme son titre l'indique, deux parties à peu près égales consacrées d'un côté à la philosophie, de l'autre à l'histoire. On y retrouve des analyses d'ouvrages récents

21. Bibliographie der Sowjetischen Philosophie. Bibliograpby of Soviet Pbilosophy, 1947-1966, Dordrecht, D. Reidel, 1959-1968, 7 volumes.

22. Philosophical Books, Leicester, Leicester University Press, vol. 1, 1960-

23. Philosophy and History; a Review of German Language Research Contributions on Pbilosophy, History and Cultural Developments (German Studies, Section 1), Tübingen, vol. 1, 1968- 
publiés en langue allemande. La particularité de ce périodique réside dans le fait que les comptes rendus sont tous rédigés en anglais et que le titre des ouvrages est donné à la fois en anglais et en allemand. Chaque numéro regroupe environ 20 à 25 recensions d'ouvrages philosophiques. Les analyses sont tantôt critiques, tantôt purement descriptives.

La revue comprend aussi une bibliographie de quelques dizaines d'articles et de livres récents publiés en allemand. Là encore la traduction anglaise accompagne le titre allemand. À la fin de la revue, le prix des ouvrages analysés est indiqué.

Philosophy and History vise donc à promouvoir à l'extérieur de l'Allemagne la connaissance d'un certain nombre de publications philosophiques et historiques.

\section{m) Le Philosopbische Rundschau}

Le Philosopbische Rundschau ${ }^{24}$ paraît depuis 1953. Ce périodique est constitué de comptes rendus critiques très élaborés d'ouvrages qui, assez fréquemment, appartiennent à un même thème. Chaque numéro comporte aussi des analyses plus courtes de livres récents. La couverture des ouvrages recensés est internationale et concerne plusieurs domaines de la philosophie. Les recensions sont toutefois rédigées en allemand. Quant aux collaborateurs, ils sont allemands pour la plupart encore que quelques-uns appartiennent à d'autres nationalités. Les noms des philosophes Hans-Georg Gadamer et Helmut Kuhn ont été étroitement liés à l'élaboration de cette revue.

Philosophische Rundschau publie en outre, de temps à autre, un supplément sur un sujet particulier. C'est ainsi qu'en 1976 un supplément de plus de 700 pages portait sur la contribution des philosophes français aux études platoniciennes.

Signalons enfin que la revue est pourvue d'un index des auteurs d'ouvrages recensés et cités et d'un index des auteurs qui signent les comptes rendus.

24. Pbilosophische Rundschau ; eine Zeitschrift für Philosophische Kritik, Tübingen, Mohr, vol. 1, 1953- 
n) Philosophischer Literaturanzeiger

Le Philosophischer Literaturanzeiger ${ }^{25}$ paraît depuis 1949, conjointement avec le Zeitschrift für philosophische Forschung. Chacun des six numéros annuels contient 15 à 20 analyses critiques d'ouvrages récemment parus en philosophie. Les analyses signées portent sur des livres en majorité allemands. Le compte rendu lui-même est toujours rédigé en allemand. Chaque numéro signale en outre quelques dizaines d'ouvrages récemment parus. La liste des ouvrages recensés apparaît dans le dernier numéro de l'année. On trouve aussi un index cumulatif des auteurs pour les volumes 1 à 10 dans le volume 10 paru en 1957.

0) Le Bollettino filosofico

Le Bollettino filosofico ${ }^{26}$ est une revue publiée par l'Institut de philosophie de l'Université de Padoue. Le bulletin paraît tous les mois depuis 1967. Il comprend des recensions d'ouvrages italiens ou étrangers, le sommaire d'une quinzaine de revues (incluant dans plusieurs cas le résumé de l'article) et une liste de livres reçus à la direction de la revue.

2) COMMENTAIRES ET PERSPECTIVES D'AVENIR SUR LE CONTRÔLE BIBLIOGRAPHIQUE COURANT EN PHILOSOPHIE

a) Le besoin d'un répertoire exhaustif et analytique

Le bilan que nous venons de dresser nous montre qu'il existe en philosophie de bons instruments permettant l'accès à la production philosophique courante. Toutes ces bibliographies assurent la fonction de signalement en fournissant des références bibliographiques assez complètes. De plus, plusieurs répertoires présentent un résumé objectif des documents. Enfin, quelques instruments, spécialement les derniers répertoires que nous avons décrits, ajoutent une analyse critique d'un certain nombre d'ouvrages.

Nous disposons donc d'une information importante qui est par surcroît largement diffusée au sein d'une quinzaine de

25. Philosophischer Literaturanzeiger, Meisenheim/Glan, Verlag Anton Hain, vol. 1, 1949-

26. Bollettino Filosofico, Padua, Universita degli studi, Instituto di Filosofia, $1967-$ 
bibliographies. Il faut bien reconnaître, néanmoins, que nous n'avons pas de bibliographie courante en philosophie qui réunirait et signalerait en un seul instrument la plupart des nouveaux documents en philosophie (livres, articles de revues, thèses, volumes de mélanges, rapports, actes de congrès, etc.) tout en présentant pour chacun d'eux une analyse objective du contenu. Cette information existe en bonne partie, mais elle est dispersée à travers plusieurs outils documentaires. Il faut, dès lors, parcourir de nombreux fascicules de diverses bibliographies courantes pour recueillir la documentation recherchée. « La pluralité des instruments documentaires est un bien, écrit M. Gilbert Varet. Leur excessive abondance serait un danger, si elle doit d'avance décourager le chercheur à l'idée d'un nombre presque illimité de recherches à faire. Il est précieux de savoir qu'un nombre fini d'instruments peut suffire à une prospection complète ${ }^{27}$.»

Tout en cherchant à éviter la redondance inutile de l'information, il faut reconnaître que pour répondre à certains besoins, nationaux ou autres, une même documentation peut se retrouver dans deux ou plusieurs instruments de travail. L'important serait de pouvoir compter, quand c'est nécessaire, sur un répertoire suffisamment complet.

L'élaboration d'un tel répertoire, analytique et relativement exhaustif, soulève quelques problèmes qu'il faut néanmoins chercher à surmonter. Et d'abord la notion même de bibliographie exhaustive s'avère souvent illusoire. Les publications en philosophie proviennent de langues et de pays très différents, si bien que la constitution d'une bibliographie internationale suppose un effort collectif et une collaboration de plusieurs organismes. Au surplus, quelques périodiques ne publient qu'occasionnellement un article philosophique, si bien qu'ils n'apparaissent pas dans la liste des revues dépouillées par un répertoire. D'autre part, la frontière entre la philosophie et les autres disciplines n'est pas toujours facile à tracer, ce qui a un impact sur la couverture de la bibliographie. De même aussi, le caractère plus général ou plus

27. VARET, Gilbert, Manuel de bibliograpbie pbilosopbique, Paris, P.U.F., 1956, tome 1, p. 12. 
spécialisé de la bibliographie courante est susceptible de satisfaire tel type de besoins au détriment des autres.

\section{b) Les points d'accès à la documentation}

Nous allons maintenant examiner une autre question, à savoir les points d'accès à la documentation. L'expression "points d'accès » recouvre ici le système de classification, les index ou tout autre moyen susceptible d'assurer la jonction entre la demande d'un chercheur et la documentation existante. Car le processus du repérage de la documentation emprunte le même parcours que le procédé d'indexation des documents mais en sens inverse. Dans le premier cas en effet, il s'agit d'établir le profil d'une recherche, d'abord au niveau conceptuel, puis au plan de la traduction des concepts en descripteurs. Le processus de l'indexation consiste à élaborer le profil des documents au niveau conceptuel et au plan de la traduction des concepts en descripteurs. C'est la comparaison entre le profil de la recherche et le profil des documents qui révèle la documentation pertinente sur un sujet.

Encore faut-il que les profils soient suffisamment détaillés et raffinés pour permettre de retrouver toute la documentation désirée. Il peut exister une documentation utile pour une recherche, mais cette documentation peut très bien ne pas être repérable, faute d'un vocabulaire adéquat.

Un répertoire bibliographique s'avère d'autant plus utile qu'il comporte des points de repère adaptés aux différents besoins des chercheurs. À cet égard, on peut distinguer des besoins documentaires généraux et des besoins documentaires particuliers. Un répertoire bibliographique courant doit s'adresser au professeur qui désire s'informer des nouvelles publications liées à son champ d'enseignement, par exemple la logique symbolique, la philosophie des sciences ou l'esthétique. Mais un répertoire doir s'adresser également au chercheur désireux de connaître les publications d'un auteur, les études sur un philosophe ou les publications sur un thème particulier comme la métaphore, la justice selon Platon ou les rapports entre l'ironie et l'humour. Par ailleurs un usager, qu'il s’intéresse à un domaine général ou à un point précis, peut 
vouloir se tenir au courant ou encore effectuer une recherche rétrospective. Les bibliographies courantes ont principalement pour objectif la mise au courant, mais on peut s'en servir aussi pour une recherche rétrospective, spécialement quand une bibliographie courante est pourvue d'un index cumulatif ou qu'elle est constituée en banque automatisée.

Les bibliographies courantes qui existent présentement en philosophie permettent le repérage de la documentation par le nom des auteurs. Certaines bibliographies, comme le Répertoire bibliographique de la philosopbie, signalent en outre les personnes qui ont contribué à une publication (éditeur intellectuel, préfacier, traducteur, etc.).

Outre l'accès à la documentation par le biais des auteurs, les bibliographies courantes en philosophie font appel à un système de classification qui permet de regrouper les publications sous des rubriques relativement générales. Dans la plupart des cas, comme nous l'avons constaté antérieurement, les livres et les articles de revues sont rangés sous l'une ou l'autre des périodes de l'histoire de la philosophie ou à l'intérieur des grandes branches de cette discipline. "The classification systems in . . . bibliographies of the century, note Michael Jasenas, are mostly curriculum-centered, at least in so far as their main divisions are concerned, because the latter represent the major branches or subjects of philosophy taught at the universities ${ }^{28}$. "

En ce qui concerne l'accès à la documentation par le biais d'un sujet spécifique, nous constatons qu'il est possible dans quelques bibliographies, notamment le Bulletin signalétique, le Philosopher's Index et la Bibliographie Pbilosophie. Cette tendance ira sans doute en s'accentuant.

L'utilisation de descripteurs plus généraux ou plus spécifiques entraîne des conséquences dont il faut tenir compte. "In general, écrit Lancaster, if we create large classes we make it easier to find all the documents on a particular topic (i.e. achieve a high recall) but more difficult to retrieve only relevant documents (i.e. achieve a high precision). Conversely,

28. JasENAS, Michael, A History of the Bibliograpby of Philosophy, p. 123. 
if we create many very small classes we would expect to be able to achieve high precision, but we may find it rather more difficult to conduct a comprehensive search to achieve high recall ${ }^{29}$."

Il reste toujours possible d'utiliser un ou deux descripteurs généraux et plusieurs mots clefs spécifiques. De toute façon, il est fréquent aujourd'hui d'attribuer plusieurs descripteurs à un document plutôt qu'un seul ou deux. L'index de la Revue philosophique de Louvain ${ }^{30}$ illustre bien ce fait.

Cette multiplicité de descripteurs s'accorde bien avec le principe de la postcoordination qui joue un rôle important dans les systèmes documentaires contemporains. En vertu de ce principe les concepts, représentés par autant de descripteurs ou de classes, sont reliés au moment de la recherche documentaire plutôt qu'au moment de l'indexation des documents. Cette méthode est particulièrement utilisable dans une recherche documentaire par ordinateur. C'est cet aspect que nous allons maintenant brièvement considérer.

\section{c) La recherche de documentation par ordinateur}

Le fait nouveau de la recherche documentaire, depuis quelques années, c'est le repérage de l'information par ordinateur. On le doit notamment aux progrès remarquables réalisés dans le domaine de l'informatique et des télécommunications. Reliées, ces deux techniques ont donné naissance à la téléinformatique.

Ce qui frappe d'abord dans le recours à l'informatique, c'est sans doute la rapidité avec laquelle on obtient l'information désirée, spécialement quand une interrogation se fait en mode conversationnel. Plus déterminante encore est peut-être la possibilité de coordonner les termes d'une recherche selon les relations de conjonction ( $\mathrm{A}$ et $\mathrm{B})$, de disjonction ( $\mathrm{A}$ ou $\mathrm{B}$ ) ou d'exclusion (A sans $B$ ) et d'atteindre ainsi une plus grande précision. L'utilisation de la logique booléenne n'est pas la

29. LANCASTER, F.W., Vocabulary Control for Information Retrieval, Washington, D.C., Information Resources Press, 1972, p. 1.

30. Register der Revue philosophique de Lowvain 44/68, 1946-1970, Liechtenstein, Kraus Reprint, 1973. 
seule méthode possible, mais c'est sans doute la plus répandue. Il existe d'autres techniques, telles que les valeurs de pondération des termes ${ }^{31}$.

La possibilité, lorsqu'elle existe dans une banque d'information, d'utiliser le vocabulaire libre en plus du vocabulaire contrôlé, accroît aussi la précision et la pertinence des références obtenues. En pareil cas nous pouvons demander n'importe quel terme, même si ce terme n'est pas contenu dans le thesaurus. Un thesaurus ne comprend pas tous les concepts d'une discipline, mais ceux qui sont le plus fréquemment utilisés. Le recours au vocabulaire libre n'élimine pas l'usage du vocabulaire contrôlé. Il le complète plutôt.

Il existe plusieurs autres avantages à la recherche de documentation par ordinateur. Souvent les banques d'information bibliographique signalent plus rapidement les données que les index imprimés. Ce facteur est important, surtout si un répertoire paraît tous les trimestres plutôt que chaque mois. D'autre part, il est possible par ordinateur d'effectuer une recherche rétrospective couvrant $5,10,15$ ans ou plus selon les banques. Or, c'est justement là une des lacunes de nos bibliographies courantes en philosophie. Dans la majorité des cas en effet, elles sont dépourvues d'index cumulatifs. Enfin, les données pertinentes à une recherche documentaire automatisée peuvent être imprimées en différé ou en conversationnel, ce qui élimine plusieurs démarches fastidieuses.

Quelle est la situation des bibliographies courantes en philosophie, compte tenu de ces nouvelles possibilités techniques que nous venons d'esquisser ? Comme nous l'avons déjà signalé antérieurement, la Bibliographie Philosophie, le Philosopher's Index et le Bulletin signalétique sont produits à l'aide de l'ordinateur (constitution des index, stockage des données, etc.). Dans le cas du Bulletin Signalétique et dans celui du Philosopher's Index, le repérage de l'information peut s'effectuer par ordinateur mais en différé. Encore faut-il souligner que les éditeurs du Philosopher's Index ont entrepris des démarches en

31. Sur ce sujet, voir le chapitre "Searching without Boolean Algebra ", contenu dans l'ouvrage de F.W. LANCAster et E.G. FAYEN, Information Retrieval On-Line, Los Angeles, Melville Publishing Company, 1973, p. 256-259. 
vue de rendre possible l'interrogation de leur banque en conversationnel et ils espèrent que leur projet se concrétisera en 1979. Si tel est le cas, une interrogation en direct du Philosopher's Index permettra d'avoir accès aux livres publiés aux États-Unis entre 1940 et 1977 , aux articles publiés aux États-Unis de 1940 à maintenant et à un certain nombre d'articles publiés ailleurs qu'aux États-Unis depuis 1967.

Il n'est pas inutile de signaler aussi que le Social Sciences Citation Index (SSCI) est présentement accessible en mode conversationnel. Cette banque, qui signale des articles de périodiques parus depuis 1972, intègre une cinquantaine de revues philosophiques.

Les progrès de la technologie influencent sûrement à l'heure actuelle l'exploitation des systèmes documentaires. Ces progrès surviennent au moment où déjà les systèmes documentaires se sont transformés sous l'influence de conceptions nouvelles comme le principe de la postcoordination. Les bibliographies courantes en philosophie sont de plus en plus marquées par ces changements. Compte tenu de l'importance que prend l'information documentaire de nos jours, les réalisations auxquelles nous avons fait écho dans notre bilan des bibliographies courantes en philosophie et les projets mis sur pied constituent une évolution chargée de promesses.

Université Laval 